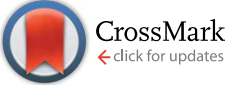

Cite this: RSC Adv., 2016, 6, 7990
Received 8th December 2015 Accepted 3rd January 2016

DOI: 10.1039/c5ra26175a

www.rsc.org/advances

\title{
A non-zipper-like tetrameric coiled coil promotes membrane fusion $\dagger$
}

\author{
Tingting Zheng, $\dot{t}^{\mathrm{a}}$ Monica Bulacu, $\dot{t}^{\mathrm{b}}$ Geert Daudey, ${ }^{a}$ Frank Versluis, ${ }^{a}$ Jens Voskuhl, ${ }^{a}$ \\ Giuliana Martelli, ${ }^{a}$ Jan Raap, ${ }^{a}$ G. J. Agur Sevink, ${ }^{c}$ Alexander Kros ${ }^{\star a}$ \\ and Aimee L. Boyle ${ }^{\star a}$
}

Two peptides, Coil-K and Coil-E, form a parallel heterodimeric coiled coil, CC-K/E, and have been shown to promote membrane fusion. This article examines the effects of reversing the sequence of Coil-E (to yield Coil-Er), on coiled-coil formation and membrane fusion. Coiled-coil assembly was studied using circular dichroism spectroscopy, paramagnetic proton NMR, fluorescence spectroscopy, analytical ultracentrifugation and computational simulations. Combined, the data show that Coil- $\mathrm{K}$ and Coil-Er combine in a 1:1 ratio to form an antiparallel tetramer, reinforcing previous studies that show small changes to peptide sequences strongly affect the stoichiometry and orientation of the resulting assemblies. Cholesterol-modified Coil- $\mathrm{K}$ and Coil-Er variants were subsequently tested for their ability to promote membrane fusion and the results were compared to the CC-K/E model system. Surprisingly, no significant differences were found between the two systems, despite the Coil-K/Er complex being 'nonzipper-like'.

\section{Introduction}

Membrane fusion occurs in all living cells where it regulates a variety of processes including fertilisation, viral infection, exocytosis, and vesicle trafficking. ${ }^{1-4}$ One of the most widely-studied fusion systems involves SNARE (soluble $N$-ethylmaleimidesensitive factor attachment protein receptor) proteins; these are a large family of proteins primarily responsible for vesicle trafficking and fusion. ${ }^{5-7}$ Whilst the exact mechanism of membrane fusion has yet to be determined, it is widely accepted that there are four steps: opposing membranes are brought into close proximity; local disruption of the membrane at the site of contact ensues; hemifusion, where outer membranes merge, occurs; and pore formation, facilitating content transfer between the two fused components, is the final result. ${ }^{8}$ SNARE proteins are located on opposing membranes and, in the first step of the fusion process, bind to form a tetrameric coiled-coil bringing the opposing membranes into close contact and triggering the fusion cascade. ${ }^{9}$ In an effort to mimic this system, and to increase the understanding of this complex process,

\footnotetext{
${ }^{a}$ Supramolecular and Biomaterials Chemistry, Leiden Institute of Chemistry, Leiden University, P.O. Box 9502, 2300RA, Leiden, The Netherlands. E-mail: a.l.boyle@ chem.leidenuniv.nl; a.kros@chem.leidenuniv.nl

${ }^{b}$ Culgi BV, Galileiweg 8, 2333BD, Leiden, The Netherlands

${ }^{c}$ Solid State NMR, Leiden Institute of Chemistry, Leiden University, P.O. Box 9502, 2300RA, Leiden, The Netherlands

$\dagger$ Electronic supplementary information (ESI) available. See DOI: 10.1039/c5ra26175a

\$ These authors contributed equally.
}

numerous models for fusion have been created using a range of molecules including DNA, peptides, and organic small molecules as fusogens. ${ }^{10-18}$ One such model system, developed in our lab, takes inspiration from SNARE proteins and is based on the interaction between two coiled-coil forming peptides Coil-K $\left([\mathrm{KIAALKE}]_{3}\right)$ and Coil-E ([EIAALEK $\left.]_{3}\right)$. These two peptides are designed to assemble into a parallel heterodimeric coiled coil (CC-K/E). ${ }^{19}$ Lipidated conjugates of Coil-K and Coil-E have been synthesised and incorporated into liposomes and, upon mixing, a parallel coiled coil is formed which forces the two opposing liposome membranes into close proximity resulting in fusion. ${ }^{20-23}$

It has been postulated that, for fusion to occur, the fusogens need to be aligned in a parallel orientation allowing them to 'zipper' up from their distal N-termini to their membraneproximal C-termini, generating an inward force that pulls the two membranes together and forces them to fuse. ${ }^{24,25}$ To probe this 'zipper' hypothesis, peptides that are anchored into the membrane at opposing termini, ${ }^{26}$ or peptides that have an antiparallel orientation can be used, Scheme 1. Previous studies have shown that both antiparallel SNARE derivatives and short antiparallel coiled coils cannot induce fusion. ${ }^{27,28}$ Intrigued by this, we endeavoured to discover whether non-zipper-like fusion was possible using antiparallel coiled coils incorporated into our membrane fusion system.

We hypothesized that, by reversing the amino acid sequence of either Coil-E or Coil-K we would generate an antiparallel coiled-coil. We were aware that changes to the core packing of coiled-coil structures can have large effects on the resulting 
(A)

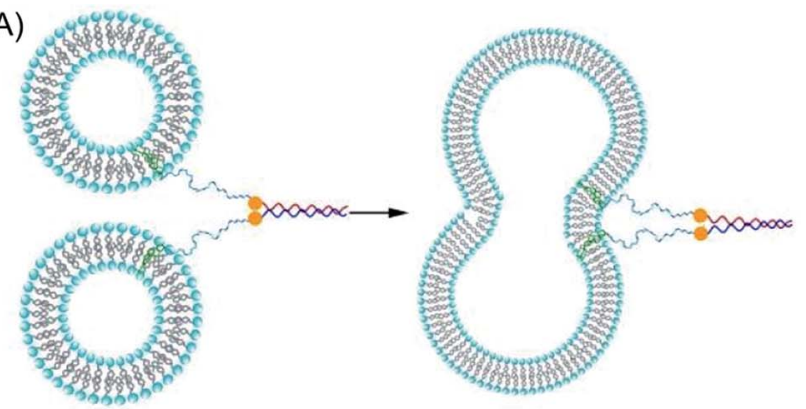

(B)
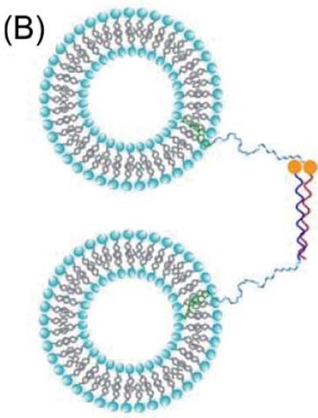

(C)

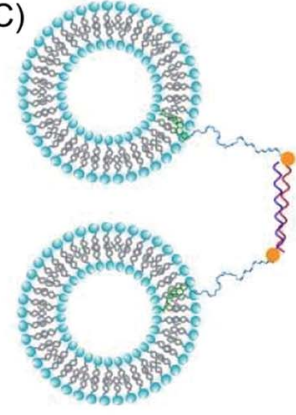

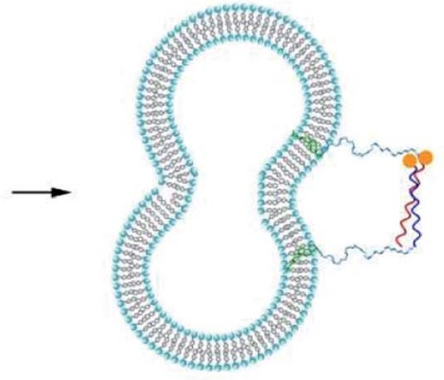
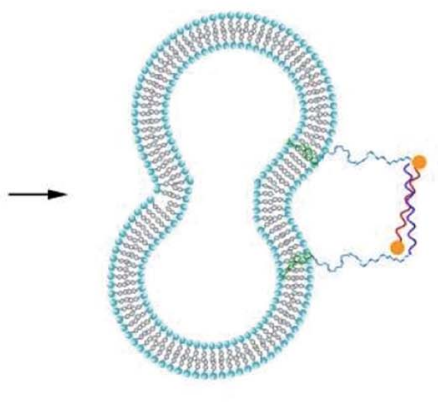

Scheme 1 Schematic representations of: (A) a zipper-like orientation of $\mathrm{CC}-\mathrm{K} / \mathrm{E}$; (B) the non-zipper-like orientation of $\mathrm{CC}-\mathrm{K} / \mathrm{E}$ resulting from anchoring coiled coils in the membrane via opposing peptide termini and; (C) the proposed non-zipper-like orientation of CC-K/Er, a putative antiparallel coiled coil. Key: red helix $=$ Coil-K, blue helix $=$ Coil-E, purple helix $=$ Coil-Er. Orange circles represent the position of the $\mathrm{N}$-terminus on each peptide.

assemblies: a well-known study of the GCN4 leucine zipper, an archetypal coiled coil, demonstrated that the oligomerisation state could be changed by altering the amino acids that comprise the hydrophobic core. ${ }^{29} \mathrm{~A}$ similar study reversed the entire GCN4 sequence, forming an antiparallel homotetramer; the native GCN4 is homodimeric. ${ }^{30}$ The reasons for these changes in oligomer state have been explained by the fact that different hydrophobic residues pack together in different manners at different positions within the coiled coil, meaning different oligomer states are necessary to facilitate the preferred packing of the different residues. ${ }^{31,32}$

With this in mind we decided to reverse the sequence of Coil$\mathrm{E}$, resulting in Coil-Er, and we analysed the resulting CC-K/Er complex using a variety of techniques including circular dichroism (CD) spectroscopy, paramagnetic proton nuclear magnetic resonance (NMR) spectroscopy, fluorescence spectroscopy, analytical ultracentrifugation, and computational

simulations to determine the orientation and oligomer state of the assembly. Finally, the fusogenicity of cholesterol-modified variants of Coil-K and Coil-Er were compared to our CC-K/E model system to determine whether the CC-K/Er system was capable of effecting fusion and, if so, whether the rate and extent of fusion was affected by employment of this antiparallel, non-zipper-like coiled-coil.

\section{Materials and methods}

\section{Materials}

Fmoc-protected amino acids, rink amide resin, and HCTU (O(1H-6-chlorobenzotriazole-1-yl)-1,1,2,2-tetramethyluronium hexafluorophosphate), were purchased from NovaBioChem. DIPEA ( $N, N$-diisopropylethylamine), piperidine, acetic anhydride, NMP ( $N$-methyl-2-pyrrolidone), DMF ( $N, N$-dimethylformamide), MeCN (acetonitrile), and TFA (trifluoroacetic acid) were obtained from Biosolve. DCM (dichloromethane), diethyl ether, $\mathrm{D}_{2} \mathrm{O}$ (deuterium oxide), TIS (triisopropylsilane), EDT (1,2-ethanedithiol), cholesterol, РуВОР (benzotriazol-1-yl-oxytripyrrolidinophosphonium hexafluorophosphate), and sulforhodamine B were obtained from Sigma Aldrich. The Fmoc$\mathrm{NH}-(\mathrm{PEG})_{12}-\mathrm{COOH}$ linker was purchased from Iris Biotech. MTSL ( $S$-(2,2,5,5-tetramethyl-2,5-dihydro-1 $H$-pyrrol-3-yl)methyl methanesulfonothioate) was obtained from Toronto Research Chemicals Inc. DOPE (1,2-dioleyl-sn-glycero-3-phosphoethanolamine), DOPC (1,2-dioleyl-sn-glycero-phosphocholine), DOPE-LR (1,2-dioleyl-sn-glycero-3-phosphoethanolamine$N$-lissamine rhodamine B sulfonyl), and DOPE-NBD (1,2dioleyl-sn-glycero-3-phosphoethanolamine- $N$-7-nitro-2-1,3benzoxadiazol-4-yl) were purchased from Avanti polar lipids.

Cholesteryl-4-amino-4-oxobutanoic acid was synthesised according to a literature procedure..$^{33}$

PBS (phosphate buffered saline) buffer contains $5 \mathrm{mM}$ $\mathrm{KH}_{2} \mathrm{PO}_{4}, 15 \mathrm{mM} \mathrm{K}_{2} \mathrm{HPO}_{4}, 150 \mathrm{mM} \mathrm{NaCl}$, pH 7.4.

\section{Methods}

Peptide synthesis. Peptides were synthesized on a CEMLiberty 1 single channel microwave peptide synthesiser using standard Fmoc chemistry. Fmoc-protected rink amide resin was employed as the solid support. All peptides were manually acetylated at the N-terminus after completion of the synthesis. Peptides without a cysteine (Cys, C) residue were cleaved from the resin and side-chain deprotected using a mixture of TFA : $\mathrm{H}_{2} \mathrm{O}:$ TIS $(95: 2.5: 2.5 \mathrm{v} / \mathrm{v}$ ) for 2 hours. Peptides with a Cys residue were cleaved from the resin with simultaneous side-chain deprotection using a mixture of TFA: EDT : $\mathrm{H}_{2}-$ $\mathrm{O}:$ TIS $(92.5: 2.5: 2.5: 2.5 \mathrm{v} / \mathrm{v})$ for 2 hours. The cleavage solution was added drop-wise into cold diethyl ether to precipitate the peptide, this was centrifuged and the liquid supernatant removed. All peptides were dried under vacuum, dissolved in deionised water and lyophilized yielding a white powder.

To form the lipidated peptide constructs CPE, CPEr, and CPK, the peptides were synthesised in the same way. After synthesis, the resin was washed with DMF. Fmoc-NH-(PEG) $)_{12}-$ $\mathrm{COOH}$ (1.1 equivalents) was subsequently activated with HCTU 
(3 equivalents) and DIPEA (4 equivalents) in DMF and, after 5 minutes, added to the resin. The coupling reaction was allowed to proceed overnight. The linker was then Fmoc-deprotected by adding a solution of piperidine (20\%) in DMF to the resin, and leaving for 15 minutes. This process was repeated three times before the resin was washed with DMF. Cholesteryl-4-amino-4oxobutanoic acid (3 equivalents) was dissolved in $2: 1(\mathrm{v} / \mathrm{v})$ DMF/DCM and activated with PyBOP (4 equivalents) and DIPEA ( 5 equivalents). After 5 minutes, this mixture was added to the resin and left overnight. The resin was then washed (DMF followed by DCM) and cleaved for 1 hour with a mixture of TFA : TIS (97.5: $2.5 \mathrm{v} / \mathrm{v})$. The lipidated constructs were then precipitated in cold diethyl ether, collected by centrifugation and dried under vacuum.

MTSL labelling. MTSL was conjugated to the peptide via a disulfide bond with a cysteine residue. Peptide $(1 \mathrm{mM})$ was dissolved in tris buffer (1 $\mathrm{M}$ tris (2-amino-2-hydroxymethylpropane-1,3-diol), $\mathrm{pH}=7.0$ ) and five equivalents of MTSL in DMF were added slowly under an argon atmosphere. The final mixture was stirred for 3 hours at room temperature. The samples were lyophilized and subsequently purified.

Peptide purification. The crude peptides were purified by RPHPLC (reversed phase-high pressure liquid chromatography), using a C18 preparative column, or a C4 column for the lipidated constructs, with a flow rate of $15 \mathrm{~mL} \mathrm{~min}^{-1}$. Peptides were eluted with a linear gradient from $\mathrm{A}$ to $\mathrm{B}$ : solvent $\mathrm{A}=0.1 \%$ TFA in $\mathrm{H}_{2} \mathrm{O}$; solvent $\mathrm{B}=\mathrm{MeCN}$. Acetylated peptides were purified using a gradient from $10 \%$ to $90 \%$ B. MTSL labelled peptides, and the lipidated peptide constructs, were purified using a gradient from $20 \%$ to $80 \%$ B. Purified peptides were lyophilized and characterized by LC-MS (liquid chromatography-mass spectrometry).

Circular dichroism spectroscopy. CD spectra were obtained using a Jasco J-815 spectropolarimeter equipped with a peltier temperature controller. The ellipticity is given as mean residue molar ellipticity, $[\theta]\left(\mathrm{deg} \mathrm{cm}^{2} \mathrm{dmol} \mathrm{res}^{-1}\right)$, calculated using the following equation: ${ }^{34}$

$$
[\theta]=\frac{100 \times[\theta]_{\mathrm{obs}}}{c n l}
$$

where $\theta_{\text {obs }}$ is the observed ellipticity (mdeg), $n$ is the number of peptide bonds, $l$ is the path length of the cuvette $(\mathrm{cm})$ and $c$ is the peptide concentration (mM).

Spectra were recorded from $260 \mathrm{~nm}$ to $190 \mathrm{~nm}$ at $25^{\circ} \mathrm{C}$. Data points were collected with a $1 \mathrm{~nm}$ bandwidth at $1 \mathrm{~nm}$ intervals, using a scan speed of $1 \mathrm{~nm} \mathrm{~s}^{-1}$. Each spectrum was an average of 3 scans. For analysis, each spectrum had the background PBS spectrum subtracted. Thermal unfolding spectra were recorded at $222 \mathrm{~nm}$, with a temperature gradient of $40{ }^{\circ} \mathrm{C}$ hour ${ }^{-1}$.

${ }^{1}$ H-magnetic resonance spectroscopy. To monitor the aromatic region of the amino acids tryptophan (Trp, W) and tyrosine (Tyr, Y); ${ }^{1} \mathrm{H}$-NMR signals in the range 6-8 ppm were recorded. The peptide amide proton signals were suppressed by proton-deuterium exchange using $\mathrm{D}_{2} \mathrm{O}$. Lyophilized peptide samples were dissolved at a concentration of $0.5 \mathrm{mg} \mathrm{mL}^{-1}$ and incubated in $\mathrm{D}_{2} \mathrm{O}$ for one hour, followed by lyophilization. This procedure was repeated three times. PBS was lyophilized and redissolved in $\mathrm{D}_{2} \mathrm{O}$ to prepare a $\mathrm{PBS} / \mathrm{D}_{2} \mathrm{O}$ buffer solution. Peptide samples were prepared with a final concentration of 0.8 $\mathrm{mM}$ in $\mathrm{PBS} / \mathrm{D}_{2} \mathrm{O}$ buffer solution. All ${ }^{1} \mathrm{H}-\mathrm{NMR}$ spectra were recorded at $25{ }^{\circ} \mathrm{C}$ on a Bruker Avance I $600 \mathrm{MHz}$ spectrometer with 32 scans for each sample.

Liposome preparation. A $1 \mathrm{mM}$ stock solution containing DOPC : DOPE : cholesterol (50: $25: 25$ mol\%) lipids in $1: 1$ (v/ v) methanol : chloroform was prepared for all fusion experiments, except for the lipid mixing experiments. For these, a 1 $\mathrm{mM}$ stock solution of DOPC : DOPE : cholesterol : DOPELR : DOPE-NBD (49.5: $24.75: 24.75: 0.5: 0.5$, mol\%) in $1: 1$ methanol : chloroform was made. Lipidated peptides were dissolved in a $1: 1(\mathrm{v} / \mathrm{v})$ choloroform : methanol mixture, to a concentration of $50 \mathrm{uM}$. $1 \mathrm{~mol} \%$ of these lipidated peptides were mixed with the appropriate liposome solution and the solvent was removed under a stream of air. For lipid mixing experiments, the dry lipid/peptide layer was rehydrated with PBS. For content mixing experiments, PBS buffer containing 20 $\mathrm{mM}$ sulphorhodamine B was added to the dried lipid/peptide mixtures containing Coil-E or Coil-Er. These solutions were subsequently sonicated for 5 minutes to yield $\sim 100 \mathrm{~nm}$ diameter liposomes. The solutions were used without further purification, with the exception of the sulforhodamine B containing liposomes, which were purified using a Sephadex column to remove any non-encapsulated sulforhodamine B.

Fluorescence spectroscopy. Lipid- and content-mixing experiments were performed on a TECAN Infinite M1000 PRO fluorimeter using a 96-well plate at $25{ }^{\circ} \mathrm{C}$. For the lipid-mixing experiments, fluorescence intensity $(F(t))$ was monitored for 1 hour by continuously measuring NBD emission at $530 \mathrm{~nm}$ upon mixing equimolar amounts of fluorescent K-liposomes with non-fluorescent E- or Er-liposomes. $F_{\max }$ was determined by using liposomes which contained half the concentration of fluorescent lipids. $F_{0}$ was determined by measuring NBD emission of $\mathrm{K}$ liposomes to which an equal amount of PBS was added. The percentage of fluorescence increase $(\% F)$ was calculated as:

$$
\% F=\left(F(t)-F_{0}\right) /\left(F_{\max }-F_{0}\right)
$$

For the content mixing experiments, the sulphorhodamine B fluorescence intensity at $580 \mathrm{~nm}$ was monitored in a continuous fashion for 1 hour, after mixing non-fluorescent K-liposomes with sulforhodamine B loaded E- or Er-liposomes. $F_{0}$ was obtained by measuring emission of sulforhodamine B-loaded E- or Er-liposomes, and $F_{\max }$ was obtained by measuring the emission of plain liposomes loaded with $10 \mathrm{mM}$ sulforhodamine B.

The FRET experiments were conducted using the same apparatus. Excitation and emission slits were set to $5 \mathrm{~nm}$. Emission spectra were measured from $290 \mathrm{~nm}$ to $450 \mathrm{~nm}$ in 1 $\mathrm{nm}$ steps at a fixed excitation wavelength of $275 \mathrm{~nm}$. The temperature was set at $25{ }^{\circ} \mathrm{C}$. The spectra were corrected by subtraction of the background spectrum. The total peptide concentration was $50 \mu \mathrm{M}$.

Sedimentation equilibrium analytical ultracentrifugation. Sedimentation equilibrium analytical ultracentrifugation 
measurements were conducted using a Beckman-Optima XL-I analytical ultracentrifuge fitted with an An-60 Ti rotor. Peptide solutions were prepared in PBS buffer, $\mathrm{pH}$ 7.4, at peptide concentrations which gave an initial absorbance in the range 0.2-0.6 A.U. The samples were spun at three speeds (between $34000-50000 \mathrm{rpm}$ ) at $20{ }^{\circ} \mathrm{C}$. The data were then fitted to a single-ideal species model using Ultrascan. ${ }^{35}$

Molecular simulations. To address the time- and lengthscales associated with coiled-coil formation, coarse-grained molecular dynamics (CGMD) simulations were carried out with the GROMACS molecular dynamics package using version 2.1 of the MARTINI coarse-grained force field and its extension to proteins. ${ }^{36-38}$ Using this model, the association behaviour of several proteins have been simulated previously, and the computational results show good agreement with previous atomistic simulations or experiments. ${ }^{39-41}$ The self-assembly process of Coil-Er and Coil-K peptides was characterised by performing 20 independent simulations for each setup, containing either one, two, or three copies of both Coil-K and CoilEr peptides, which were initially randomly distributed in solvent (water with $\mathrm{Na}^{+}$and $\mathrm{Cl}^{-}$ions). The starting coarsegained structures for Coil-K have been mapped from the 20 NMR-derived atomistic models of a near identical peptide reported by Hodges. ${ }^{42}$ The structures for Coil-Er were mapped from 20 different coordinate frames obtained by atomistic simulations of the peptide in solution, for an induced $\alpha$-helical secondary structure. Before carrying out the simulations, steepest descent minimization was performed, followed by relaxation of the solvent (positional restraints on the whole peptide) and of the side chain beads (positional restraints on the peptide backbone). After this, the system was further simulated without any restraints for at least 40 microseconds to allow self-assembly and equilibration of the formed supramolecular structures. A detailed description of the model and the simulation conditions is given in the electronic ESI. $\dagger$

\section{Results and discussion}

\section{Peptide design and synthesis}

In this study, Coil-K retained its original sequence, with the addition of a Trp at the C-terminus to allow for quantification and analytical studies. A glycine (Gly, G) was introduced to act as a spacer between the chromophore and the coiled-coil forming portion of the sequence. This gave rise to a Coil-K variant with the sequence: (KIAALKE) ${ }_{3} \mathrm{GW}$. Coil-E was redesigned to possess the reversed amino acid sequence, yielding Coil-Er. A Tyr residue was attached to the N-terminus, again to allow for quantification and analytical studies, whilst a Gly spacer was also included. This resulted in Coil-Er having the sequence: YG(EIAALEK) $)_{3}$.

Additionally, the paramagnetic nitroxyl radical MTSL ( $S$-(1-oxyl-2,2,5,5-tetramethyl-2,5-dihydro-1 $H$-pyrrol-3-yl)methyl methanesulfonothioate) was conjugated to Coil-K via a cysteine residue. Two MTSL-labelled derivatives were generated: attachment at the C-terminus yielded Coil-K*; whereas conjugation to the $\mathrm{N}$-terminus produced Coil-* K. This label offered the opportunity to investigate the stoichiometry and relative peptide orientation of $\mathrm{CC}-\mathrm{K} / \mathrm{Er}$ by paramagnetic proton NMR and steady-state fluorescence measurements. ${ }^{43}$

Peptides were synthesised on Rink amide resin using standard Fmoc solid-phase synthesis techniques and purified by reversed-phase HPLC. Peptides were identified using LC-MS mass spectrometry, and analytical HPLC confirmed the purity of the peptides was $>99 \%$ (ESI Fig. S1 and S2 $\dagger$ ).

\section{Circular dichroism spectroscopy}

Circular dichroism (CD) spectroscopy was used to probe the secondary structure of the peptides. At room temperature, both Coil-K and Coil-Er are largely unfolded, exhibiting only small amounts of $\alpha$-helical character, (Fig. 1A). The equimolar mixture, in contrast, is well folded as an $\alpha$-helical assembly and exhibits typical coiled-coil characteristics with the $[\theta] 222 \mathrm{~nm} /[\theta]$ $208 \mathrm{~nm}$ ellipticity ratio $>1$. $^{\mathbf{4 4}}$

The binding stoichiometry of Coil-K to Coil-Er was subsequently determined. A Job-plot was created by changing the ratio of the mole fraction of Coil-K and Coil-Er, while keeping the total peptide concentration constant. A minimum molar ellipticity at $[\theta] 222 \mathrm{~nm}$ was observed when Coil-K and Coil-Er were mixed in equimolar amounts, indicative of a $1: 1$ binding stoichiometry, (Fig. 1B).

Finally, the thermal stability of CC-K/Er was investigated. A solution containing a $1: 1$ mixture of Coil-K and Coil-Er was heated from $5{ }^{\circ} \mathrm{C}$ to $95{ }^{\circ} \mathrm{C}$ and a sigmoidal curve, indicative of a two-state transition from a folded to an unfolded complex was observed, (Fig. 1C). The melting temperature $\left(T_{\mathrm{m}}\right)$ was found to be $64{ }^{\circ} \mathrm{C}$, similar to the $T_{\mathrm{m}}$ of $57^{\circ} \mathrm{C}$ recorded for the $\mathrm{CC}-\mathrm{K} / \mathrm{E}$ complex. ${ }^{43}$

\section{Paramagnetic ${ }^{1} \mathrm{H}$-proton-NMR spectroscopy}

Paramagnetic NMR spectroscopy was used to confirm the stoichiometry of the CC-K/Er complex, and to explore the relative orientation of the peptides within the complex.

Our previous work demonstrated that these properties can be facilely determined by examining whether suppression of the peptide-specific proton signals occurs due to the paramagnetic relaxation enhancement (PRE) effect. ${ }^{\mathbf{4 3 , 4 5 , 4 6}}$ Full suppression of the proton signals occurs within a $10.5 \AA$ radius of the paramagnetic center, and partial suppression occurs within $13 \AA^{4{ }^{47}}$ To observe such an effect, peptides need to possess a functional group with a characteristic NMR signal, and one of the peptides is required to incorporate a signal suppressing paramagnetic spin label. Aromatic proton signals are well separated from other proton signals, therefore the signals from Trp and Tyr were monitored. In addition, the paramagnetic spin label MTSL was introduced onto either the $\mathrm{N}$ - or the C-terminus of Coil-K, yielding the aforementioned, Coil-* $\mathrm{K}$ and Coil- $\mathrm{K}^{*}$. To avoid spectral overlap of the aromatic protons with $\mathrm{N}-\mathrm{H}$ amide protons, the latter were suppressed by H-D exchange.

The aromatic region of Trp exhibits four multiplets while Tyr shows two doublets, (ESI Fig. S3A \& B $\dagger$ ). Peptide Coil-K* shows full suppression of the Trp signals, (ESI Fig. S3C $\dagger$ ), as the average distance between Trp and the MTSL label is 6.6 A. In contrast, in peptide Coil-* $\mathrm{K}$ the average distance between the 

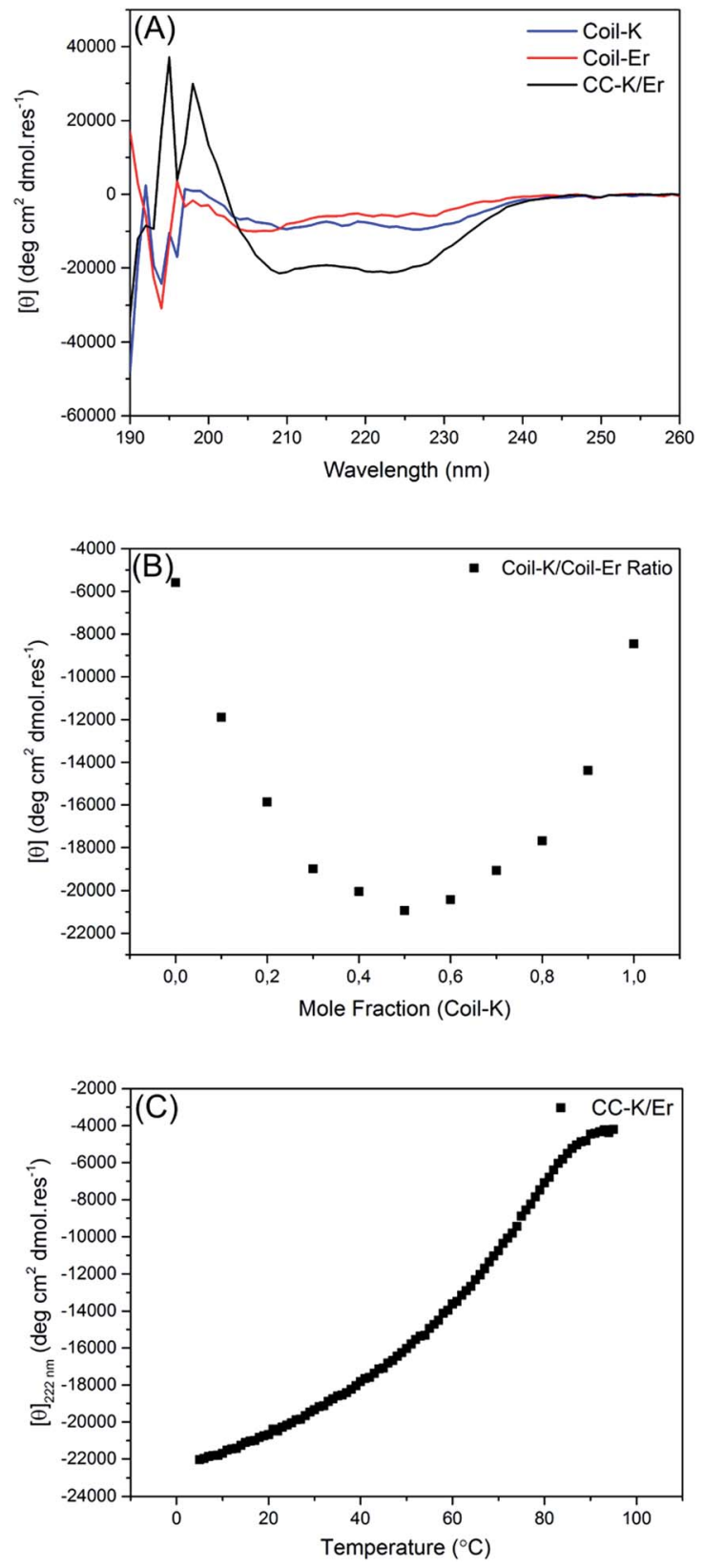

Fig. 1 Circular dichroism spectra of Coil-K and Coil-Er. (A) CD spectra of Coil-K, Coil-Er, and $1: 1 \mathrm{CC}-\mathrm{K} / \mathrm{Er}$. (B) Mean residue molar ellipticities at $222 \mathrm{~nm}$ for mixtures of Coil- $\mathrm{K}$ and Coil-Er as a function of the mole fraction of Coil-K. [Total peptide] $=100 \mu \mathrm{M}, 5^{\circ} \mathrm{C}$, PBS pH 7.4. (C) Thermal unfolding curve, monitored at $222 \mathrm{~nm}$, of the $1: 1 \mathrm{CC}-\mathrm{K} / \mathrm{Er}$ complex; the sigmoidal shape is indicative of a two-state unfolding transition. [Total peptide] $=100 \mu \mathrm{M}, \mathrm{PBS}, \mathrm{pH} 7.4$.

aromatic protons and the MTSL label is $36.7 \AA$, and therefore no PRE effect is observed, (ESI Fig. S3D $\dagger$ ).

When Coil-K and Coil-Er are mixed in a $1: 1$ ratio, all aromatic proton signals are observed, (Fig. 2A, black line). Similarly, when Coil-*K and Coil-Er are mixed, all aromatic proton signals are observed, hence there is no PRE effect (Fig. 2A, olive green line). When Coil-Er is combined with Coil$\mathrm{K}^{*}$ however, suppression of both Trp and Tyr signals are observed (Fig. 2A, lime green line), indicating that both residues
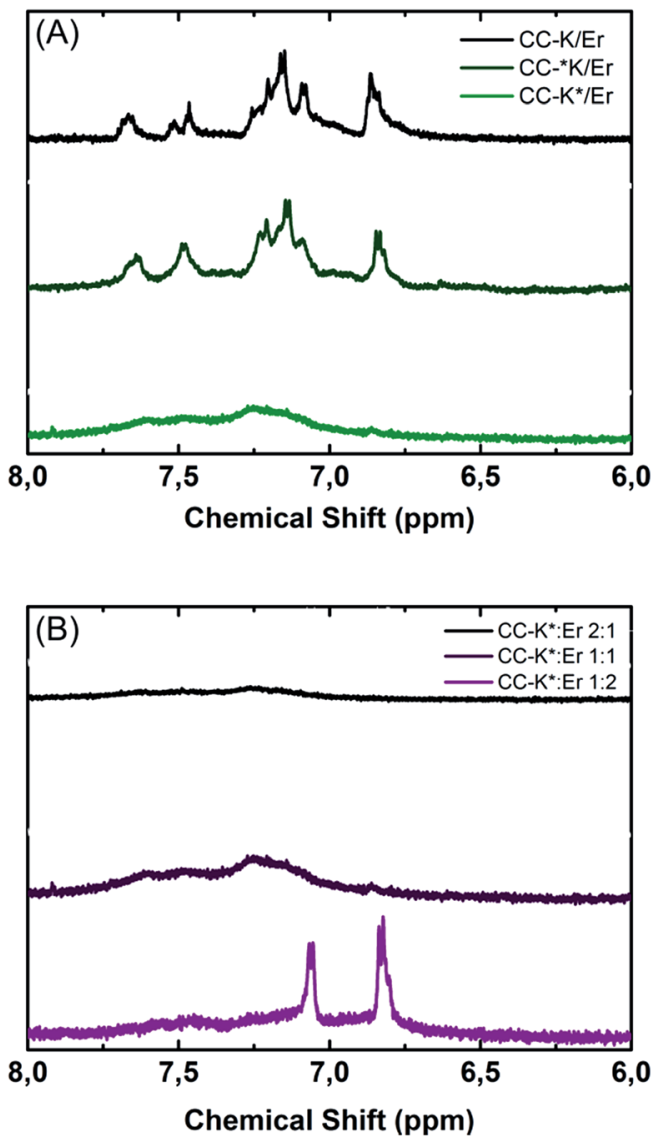

Fig. 2 Aromatic region (6-8 ppm) of ${ }^{1} \mathrm{H}-\mathrm{NMR}$ spectra showing Trp indole and Tyr hydroxyphenyl functional groups of CC-K/Er. (A) Aromatic signals of CC-K/Er. Black line: CC-K/Er; olive green line: CC*K/Er; and lime green line: CC-K*/Er. (B) Aromatic signals of CC- $\mathrm{K} *$ : Er at different molar ratios. Black line: $2: 1$ ratio; purple line: $1: 1$ ratio; magenta line $1: 2$ ratio. [Total peptide] $=0.8 \mathrm{mM}, \mathrm{PBS}, \mathrm{pH} 7.4,25^{\circ} \mathrm{C}$.

are within $10.5 \AA$ of the MTSL label. This clearly demonstrates that the peptides are assembled in an anti-parallel orientation, as the distance between Tyr and the MTSL label is >13.5 $\AA$ in the parallel orientation.

As CD spectroscopy indicated that Coil-K and Coil-Er bind in a 1:1 stoichiometry, NMR experiments were performed to confirm this. When Coil-K* and Coil-Er were mixed in a $1: 1$ molar ratio (Fig. 2B, black line), the Tyr proton signals were fully suppressed. This was also observed for a $2: 1$ (Coil-K* : Coil-Er) mixture (Fig. 2B, purple line). However, when Coil-K* and CoilEr were mixed in 1:2 molar ratio, Tyr proton signals were visible, (Fig. 2B, magenta line). This indicates there is an excess of Coil-Er which is not suppressed, and supports the conclusions drawn from the CD experiments that the two peptides bind in a $1: 1$ stoichiometry.

\section{Fluorescence spectroscopy}

To confirm the relative peptide orientation, FRET (fluorescence resonance energy transfer) experiments were performed. FRET occurs when the donor and acceptor are within the Förster distance $(10 \AA) .{ }^{48}$ An equimolar mixture of Coil-K 
and Coil-Er was examined, and it revealed that the Coil-K fluorescence intensity increased while the Coil-Er fluorescence intensity decreased, Fig. 3. This is expected when CoilEr and Coil-K form coiled coils with an antiparallel orientation, allowing FRET between the donor Tyr, and the acceptor Trp. This confirms the distance between the N-terminus of peptide Coil-Er and the C-terminus of peptide Coil-K is $<10 \AA$, confirming an antiparallel orientation of CC-K/Er, which is consistent with the findings of the NMR experiments, and confirmed our hypothesis that reversing one of the peptide sequences would result in an antiparallel orientation of the resulting coiled coil.

\section{Analytical ultracentrifugation}

To determine the oligomer state of the CC-K/Er species in solution, analytical ultracentrifugation (AUC) sedimentation equilibrium measurements were performed. Coil-K and Coil-Er were analysed in a 1:1 ratio as both the CD and NMR data indicated the two peptides bind in a $1: 1$ stoichiometry. The collected datasets fitted well to a single-ideal species model, (ESI Fig. S6 $\dagger$ ), and the average mass of the species was determined to be $10620 \mathrm{Da}$, with 95\% confidence limits (determined by Monte Carlo analysis) found to be $+57 \mathrm{Da},-64 \mathrm{Da}$. Hence, weight-averaged molecular weights indicating a tetrameric species were observed.

The fact that Coil-K/Er forms a tetrameric species is unexpected, as previous studies have noted it forms a dimer, although no analytical studies were conducted to corroborate these claims. ${ }^{49}$ On reflection, the fact that a tetramer is formed is not surprising; this is in line with findings from previous studies of the GCN4 coiled coil which indicated that altering the residues that comprise the hydrophobic core also affects the oligomerisation state of the resulting assembly, ${ }^{29,30}$ although it should be noted such studies were conducted with

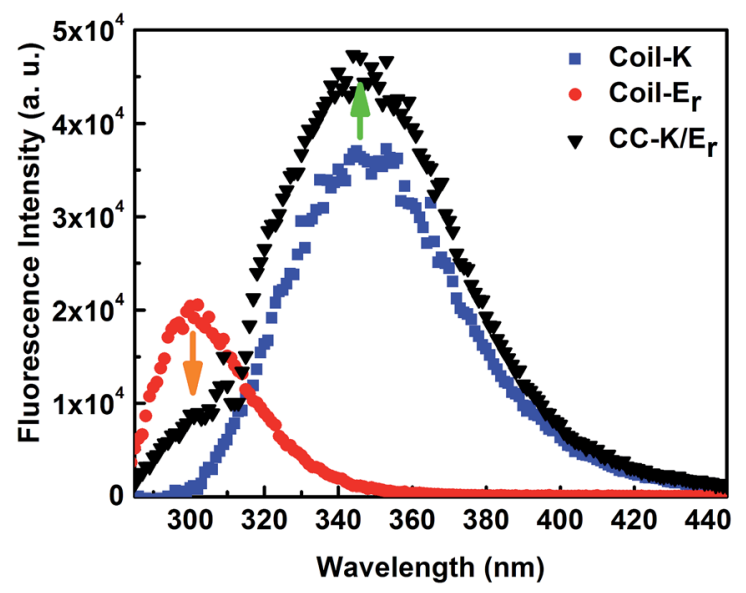

Fig. 3 Fluorescence emission spectra of Trp and Tyr on peptides Coil$K$ and Coil-Er. Blue line: Coil-K; red line: Coil-Er; black line: 1 : 1, CC-K/ Er. The green arrow indicates the increase in Trp signal, and the orange arrow shows the decrease of the Tyr signal that is observed when the peptides are mixed in a $1: 1$ ratio. [Total peptide] $=50 \mu \mathrm{M}, \mathrm{PBS}, \mathrm{pH}=$ $7.4,25^{\circ} \mathrm{C}$. homodimeric species; similar experiments with heteromeric sequences have not, to our knowledge, been conducted.

\section{Molecular simulations}

Collectively, the experimental data strongly indicate that Coil-K and Coil-Er associate in a 1:1 ratio to form an antiparallel tetramer. However, the exact arrangement of the peptides within this tetramer cannot be determined without obtaining a crystal structure. Coarse-grained molecular dynamics (CGMD) simulations were performed to obtain additional information about the preferred orientations of the peptides within the tetramer. In addition, simulations of 6 peptides in solution, (results not shown), i.e. containing 3 copies of both Coil-K and Coil-Er, showed the formation of a dimer and a tetramer indicating the tetramer is indeed the preferred structure. Formation of a trimer was not observed during the simulations.

Focussing on the tetramer simulations, two copies of both Coil-K and Coil-Er were initially placed in an $11 \mathrm{~nm}^{3}$ simulation box that also contained explicit solvent (water and $\mathrm{Na}^{+} / \mathrm{Cl}^{-}$ions) to mimic experimental conditions. The simulations were run for $40 \mu \mathrm{s}$, after which time the formation pathways and resulting assemblies were analysed.

In all simulations, the randomly distributed peptides spontaneously aggregate into supramolecular structures. The four peptides first come into contact, and then orientate parallel to each other, forming a 4-helix-bundle with a hydrophobic core. This aggregation process is relatively fast, approximately 0.5 microseconds, while 20 to 40 microseconds are needed for further equilibration; during this stage misaligned peptides rotates around their own axis or flip to form an antiparallel structure and optimize the hydrophobic core. After equilibration, each peptide forms two longitudinal interfaces with two neighbouring peptides, and the diameter of the cylindrical assembly is $\sim 2.5 \mathrm{~nm}$.

All possible tetrameric structures can be divided into two major classes: those with four K-Er interfaces; or assemblies with two K-Er interfaces, together with one $\mathrm{K}-\mathrm{K}$ and one $\mathrm{Er}-\mathrm{Er}$ interface. Accounting for parallel or antiparallel peptide orientations, as well as symmetry, 11 distinct peptide arrangements within the tetramer are possible.

Our $40 \mu \mathrm{s}$ simulations provide the means to discriminate between these potential arrangements. From the 20 independent simulations that were performed, we identified only two stable structures, (Fig. 4). The first structure has two K-Er interfaces, along with one $\mathrm{K}-\mathrm{K}$ and one Er-Er interface. The helices at the $\mathrm{K}$-Er interfaces are antiparallel, whilst the helices at the $\mathrm{K}-\mathrm{K}$ and Er-Er interfaces are aligned in a parallel orientation, (Fig. 4A). The second structure possesses four $\mathrm{K}-\mathrm{Er}$ interfaces, with antiparallel orientations of the helices at these interfaces but parallel orientations of the two Coil-K and two Coil-E helices (Fig. 4B).

Our computation setup, which relies on extensive pathway sampling for the various starting conditions, gives us confidence that the most probable binding conformations are indeed sampled by the CGMD simulations. This is further supported by observations of a complete reorientation of the 

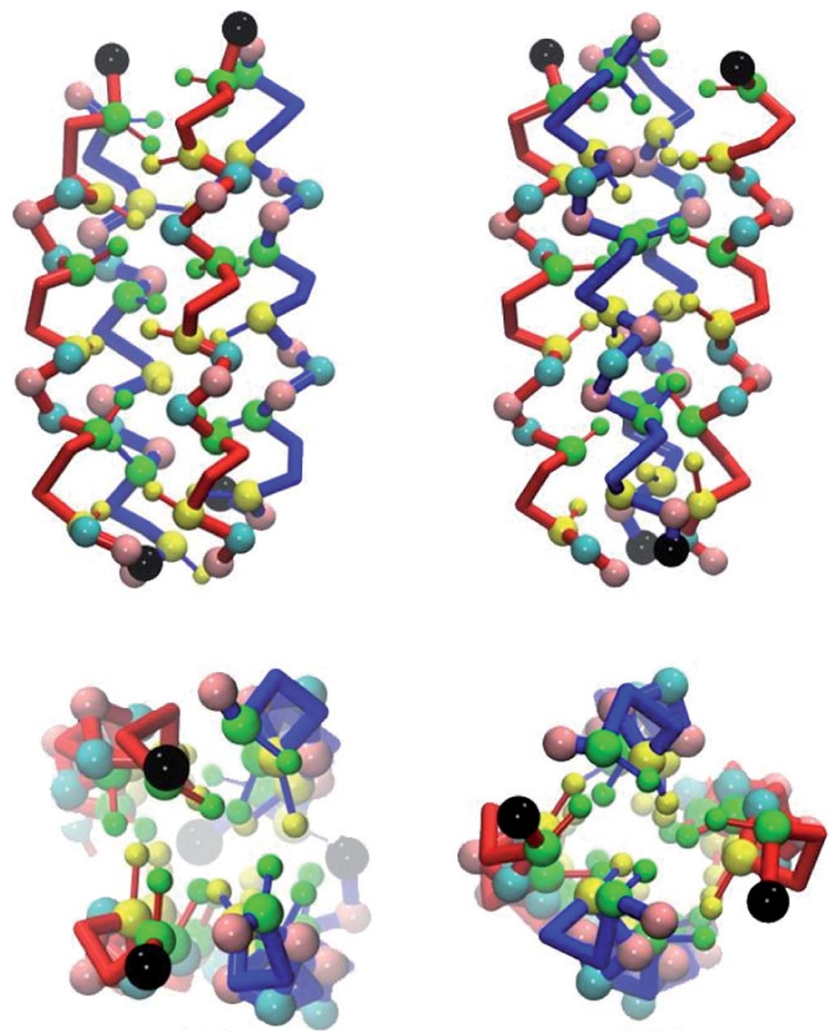

(A)

(B)

Fig. 4 The two stable CC-K/Er tetramer configurations resulting from CGMD simulations. The upper panel is the lateral view whilst the lower panel shows the top-down view. (A) A putative CC-K/Er antiparallel tetramer with two $\mathrm{K}-\mathrm{Er}$ interfaces. (B) A representation of a $\mathrm{CC}-\mathrm{K} / \mathrm{Er}$ antiparallel tetramer with four $\mathrm{K}-\mathrm{Er}$ interfaces. Key: Coil- $\mathrm{K}=$ red backbone; Coil-Er = blue backbone; green beads = isoleucine; yellow beads = leucine; pink beads = glutamic acid; cyan beads - lysine; black bead $=\mathrm{N}$-terminus.

peptides, even after association, an event that is generally considered to be rare on the timescale of molecular simulations. In the tetramer simulations no dimers were observed in the simulation time, and the only two stable structures observed has an antiparallel orientation, supporting the conclusion from the experimental evidence that an antiparallel tetrameric assembly is formed by the association of these peptides.

\section{Fusion studies}

A variety of analytical and computational techniques have shown that Coil-K and Coil-Er form an antiparallel tetramer, whereas previous studies showed that Coil-K and Coil-E form a parallel dimer.

CC-K/E is known to induce efficient and targeted membrane fusion when both peptides are modified with a lipid anchor and incorporated into liposome membranes. ${ }^{20,22,23,26}$ These fusogenic constructs comprise three distinct segments: the peptide segment, which serves as the recognition unit; a hydrophobic cholesterol anchor, which enables the construct to be secured in a liposome membrane, and; a hydrophilic linker, which is thought to be crucial for allowing force transduction and therefore helping to facilitate fusion in natural SNARE systems. ${ }^{6,50,51}$

In addition to the linking region being crucial for fusion, it is postulated that the peptides need to be in a parallel orientation, as their 'zippering' from one terminus to the other as they fold provides the force that draws the two membranes close together and induces them to fuse. ${ }^{24,25}$ Non-zipper-like coiled coils, that is, those with an antiparallel orientation should not induce fusion if it is indeed the 'zipper-like' folding of the fusogens that induces fusion.

To determine whether CC-K/Er, which assembles in a nonzipper-like manner, is able to induce fusion, cholesterolanchored constructs were generated with the peptide and cholesterol being separated by a polyethyleneglycol $(\mathrm{PEG})_{12}$ spacer. The resulting lipidated peptides were denoted CPK and CPEr. CPE was also synthesised so comparisons regarding the rate and efficiency of fusion of CC-K/E and CC-K/Er could be made. Peptide-decorated liposomes were prepared, and fusion experiments were performed by combining equimolar amounts of Coil-K liposomes with either Coil-E- or Coil-Er-containing liposomes.

Lipid-mixing studies. Lipid mixing experiments were performed to investigate the extent to which both coiled-coil motifs were able to induce lipid mixing; that is, exchange of lipids between the two liposome populations. For this assay, fluorescent DOPE-lipids (DOPE-NBD and DOPE-LR) were incorporated into the membrane of the Coil-K decorated liposomes. Upon mixing of lipids from these Coil-K liposomes with either Coil-E or Coil-Er liposomes, the average distance between DOPE-NBD (FRET donor) and DOPE-LR (FRET acceptor) increases, giving rise to an increase in donor emission. Donor emission was continuously monitored for one hour and the results show an increase in donor emission for both CC-K/E and CC-K/Er systems, (Fig. 5A), indicative of lipid mixing. Lipid mixing occurs rapidly in both systems for the first ten minutes after which time is slows significantly, this is consistent with previous studies although it should be noted that the peptides comprising the CC-K/E system used here contain Trp and Tyr residues whereas those in previous systems did not. ${ }^{22}$ Both the rate and the extent of lipid mixing is comparable in both systems, with the CC-K/Er achieving, surprisingly, a marginally greater extent of lipid mixing after one hour, (56\%), compared to the CC-K/E system (50\%).

Content-mixing assays. Full fusion is defined as the mixing of the contents of the inner aqueous compartments of the liposomes, therefore we proceeded to perform a content mixing assay to establish whether the CC-K/Er system facilitated content- as well as lipid-mixing. This was of particular interest as it has previously been demonstrated that while non-zipperlike coiled coils can induce lipid mixing, content mixing is not observed. ${ }^{49}$

For these experiments, Coil-E or Coil-Er decorated liposomes were loaded with sulforhodamine $\mathrm{B}$ at a self-quenching concentration of $20 \mathrm{mM}$. If full fusion between these liposomes and liposomes decorated with Coil-K occurs, the sulforhodamine B concentration decreases, resulting in relief of selfquenching and an increase in fluorescence intensity, which is 

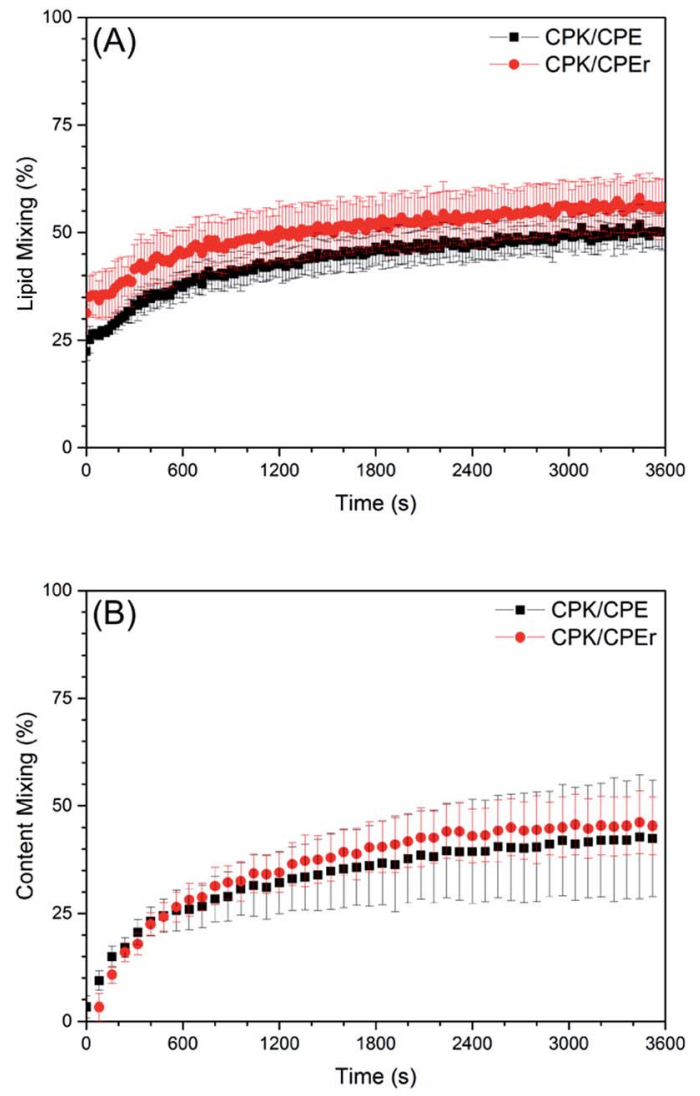

Fig. 5 Lipid- and content-mixing assays. (A) Fluorescence curves showing efficient lipid mixing between fluorescent CPK liposomes and non-fluorescent CPE and CPEr liposomes. (B) Content mixing between CPK liposomes and sulforhodamine B-containing CPE and CPEr liposomes. Key: black line $=1: 1 \mathrm{CPK}$ and CPE liposomes; red line $=1: 1$ mixtures of $C P K$ and $C P E r$ liposomes. Conditions: liposome concentration $=0.1 \mathrm{mM}$ with $1 \mathrm{~mol} \%$ peptide, $\mathrm{PBS}, \mathrm{pH} 7.4,25^{\circ} \mathrm{C}$.

indeed what was observed for both systems, (Fig. 5B). As with the lipid mixing experiments, no significant differences in the rates of content mixing were observed, and after one hour both systems demonstrated a similar extent (45\%) of content mixing.

Taken together these results indicate that, for this peptidemediated fusion system, both lipid- and content-mixing can be facilitated by zipper-like and non-zipper-like fusogens. This suggests that the way in which the peptides fold is not important, what is important is that energy is generated upon folding and that this can be transduced, presumably via the linking region, to the liposomes themselves resulting in fusion events.

\section{Conclusions}

Reversing the peptide sequence of Coil-E yields Coil-Er which, in the presence of equimolar amounts of Coil-K, results in the formation of an antiparallel tetrameric coiled coil and not an antiparallel dimeric coiled coil as previously reported. ${ }^{27}$ This shows that, in line with previous studies on proteins and peptides, reversing the peptide sequence significantly effects the orientation and oligomer state of the resulting assemblies. ${ }^{19,42,45}$ The generation of an antiparallel coiled coil offered a new 'non-zipper-like' peptide candidate for our membrane fusion model system. Cholesterol modified variants of Coil-K and Coil-Er were produced and were subsequently demonstrated to induce liposome fusion to an extent comparable to, and in the case of lipid-mixing surpassing that of, CC-K/E induced fusion. This shows that, for this peptide-mediated membrane fusion system, both zipper-like and non-zipper-like coiled-coils are capable of inducing efficient liposome fusion. Future work will focus on elucidating which elements are crucial for fusion if it is indeed not the way in which the peptides fold. As previous studies have implicated the linking region between the fusogen and the membrane anchor as being crucial for efficient fusion, ${ }^{6,50,51}$ this would be an intriguing candidate for future studies.

\section{Acknowledgements}

T. Z. acknowledges the support of the CSC. A. L. B. acknowledges the support of the FOM. M. B. and G. J. A. S. acknowledge the NCF (Netherlands Computing Facilities). A. K. acknowledges the support of the European Research Council (ERC) via an ERC starting grant (240394) which funded F. V. and G. M, and the support of the Netherlands Organisation for Scientific Research (NWO) via a ChemThem grant that funded G. D. We are grateful to Dr Hana Robson Marsden for productive discussions, and to Dr Thomas Sharp for critical reading of this manuscript.

\section{References}

1 J. E. Gerst, Cell. Mol. Life Sci., 1999, 55, 707-734.

2 M. E. Hemler, Annu. Rev. Cell Dev. Biol., 2003, 19, 397-422.

3 R. Jahn and R. H. Scheller, Nat. Rev. Mol. Cell Biol., 2006, 7, 631-643.

4 M. Kielian and F. A. Rey, Nat. Rev. Microbiol., 2006, 4, 67-76.

5 A. Maximov, J. Tang, X. F. Yang, Z. P. P. Pang and

T. C. Sudhof, Science, 2009, 323, 516-521.

6 T. C. Sudhof and J. E. Rothman, Science, 2009, 323, 474-477.

7 C. Hu, M. Ahmed, T. J. Melia, T. H. Sollner, T. Mayer and J. E. Rothman, Science, 2003, 300, 1745-1749.

8 T. Weber, B. V. Zemelman, J. A. McNew, B. Westermann, M. Gmachl, F. Parlati, T. H. Sollner and J. E. Rothman, Cell, 1998, 92, 759-772.

9 R. Jahn, T. Lang and T. C. Sudhof, Cell, 2003, 112, 519-533. 10 Y. H. Chan, B. van Lengerich and S. G. Boxer, Biointerphases, 2008, 3, FA17.

11 Y. Gong, Y. Luo and D. Bong, J. Am. Chem. Soc., 2006, 128, 14430-14431.

12 A. Kashiwada, K. Matsuda, T. Mizuno and T. Tanaka, Chemistry, 2008, 14, 7343-7350.

13 A. Kashiwada, M. Tsuboi, N. Takamura, E. Brandenburg, K. Matsuda and B. Koksch, Chem.-Eur. J., 2011, 17, 61796186.

14 A. S. Lygina, K. Meyenberg, R. Jahn and U. Diederichsen, Angew. Chem., Int. Ed., 2011, 50, 8597-8601.

15 M. M. Ma and D. Bong, Acc. Chem. Res., 2013, 46, 2988-2997. 
16 V. Marchi-Artzner, T. Gulik-Krzywicki, M. A. GuedeauBoudeville, C. Gosse, J. M. Sanderson, J. C. Dedieu and J. M. Lehn, ChemPhysChem, 2001, 2, 367-376.

17 G. Stengel, L. Simonsson, R. A. Campbell and F. Hook, J. Phys. Chem. B, 2008, 112, 8264-8274.

18 J. Voskuhl and B. J. Ravoo, Chem. Soc. Rev., 2009, 38, 495505.

19 J. R. Litowski and R. S. Hodges, J. Biol. Chem., 2002, 277, 37272-37279.

20 H. R. Marsden, N. A. Elbers, P. H. H. Bomans, N. Sommerdijk and A. Kros, Angew. Chem., Int. Ed., 2009, 48, 2330-2333.

21 H. R. Marsden, A. V. Korobko, T. T. Zheng, J. Voskuhl and A. Kros, Biomater. Sci., 2013, 1, 1046-1054.

22 F. Versluis, J. Voskuhl, B. van Kolck, H. Zope, M. Bremmer, T. Albregtse and A. Kros, J. Am. Chem. Soc., 2013, 135, 8057-8062.

23 T. T. Zheng, J. Voskuhl, F. Versluis, H. R. Zope, I. Tomatsu, H. R. Marsden and A. Kros, Chem. Commun., 2013, 49, 3649-3651.

24 Y. A. Chen and R. H. Scheller, Nat. Rev. Mol. Cell Biol., 2001, 2, 98-106.

25 R. C. Lin and R. H. Scheller, Neuron, 1997, 19, 1087-1094.

26 F. Versluis, J. Dominguez, J. Voskuhl and A. Kros, Faraday Discuss., 2013, 166, 349-359.

27 G. Pahler, C. Panse, U. Diederichsen and A. Janshoff, Biophys. J., 2012, 103, 2295-2303.

28 K. Weninger, M. E. Bowen, S. Chu and A. T. Brunger, Proc. Natl. Acad. Sci. U. S. A., 2003, 100, 14800-14805.

29 P. B. Harbury, T. Zhang, P. S. Kim and T. Alber, Science, 1993, 262, 1401-1407.

30 P. R. Mittl, C. Deillon, D. Sargent, N. Liu, S. Klauser, R. M. Thomas, B. Gutte and M. G. Grutter, Proc. Natl. Acad. Sci. U. S. A., 2000, 97, 2562-2566.

31 F. H. C. Crick, Acta Crystallogr., 1953, 6, 689-697.

32 D. N. Woolfson, Adv. Protein Chem., 2005, 70, 79-112.

33 Q. Sun, S. Cai and B. R. Peterson, Org. Lett., 2009, 11, 567570.

34 J. R. Litowski and R. S. Hodges, J. Pept. Res., 2001, 58, 477492.
35 B. Demeler, in Modern Analytical Ultracentrifugation: Techniques and Methods, ed. D. J. Scott, S. E. Harding and A. J. Rowe, Royal Society of Chemistry, Cambridge, 2005, vol. 11, pp. 210-229.

36 B. Hess, C. Kutzner, D. van der Spoel and E. Lindahl, J. Chem. Theory Comput., 2008, 4, 435-447.

37 S. J. Marrink, H. J. Risselada, S. Yefimov, D. P. Tieleman and A. H. de Vries, J. Phys. Chem. B, 2007, 111, 7812-7824.

38 L. Monticelli, S. K. Kandasamy, X. Periole, R. G. Larson, D. P. Tieleman and S. J. Marrink, J. Chem. Theory Comput., 2008, 4, 819-834.

39 D. Sengupta and S. J. Marrink, Phys. Chem. Chem. Phys., 2010, 12, 12987-12996.

40 L. V. Schafer, D. H. de Jong, A. Holt, A. J. Rzepiela, A. H. de Vries, B. Poolman, J. A. Killian and S. J. Marrink, Proc. Natl. Acad. Sci. U. S. A., 2011, 108, 1343-1348.

41 X. Periole, A. M. Knepp, T. P. Sakmar, S. J. Marrink and T. Huber, J. Am. Chem. Soc., 2012, 134, 10959-10965.

42 D. A. Lindhout, J. R. Litowski, P. Mercier, R. S. Hodges and B. D. Sykes, Biopolymers, 2004, 75, 367-375.

43 T. T. Zheng, A. Boyle, H. R. Marsden, D. Valdink, G. Martelli, J. Raap and A. Kros, Org. Biomol. Chem., 2015, 13, 1159-1168. 44 N. E. Zhou, C. M. Kay and R. S. Hodges, J. Biol. Chem., 1992, 267, 2664-2670.

45 B. Apostolovic and H. A. Klok, Biomacromolecules, 2008, 9, 3173-3180.

46 T. Gruene, M. K. Cho, I. Karyagina, H. Y. Kim, C. Grosse, K. Giller, M. Zweckstetter and S. Becker, J. Biomol. NMR, 2011, 49, 111-119.

47 C. Peggion, M. Jost, W. M. de Borggraeve, M. Crisma, F. Formaggio and C. Toniolo, Chem. Biodiversity, 2007, 4, 1256-1268.

48 J. Eisinger, B. Feuer and A. A. Lamola, Biochemistry, 1969, 8, 3908-3915.

49 K. Meyenberg, A. S. Lygina, G. van den Bogaart, R. Jahn and U. Diederichsen, Chem. Commun., 2011, 47, 9405-9407.

50 J. A. McNew, T. Weber, D. M. Engelman, T. H. Sollner and J. E. Rothman, Mol. Cell, 1999, 4, 415-421.

51 J. A. McNew, T. Weber, F. Parlati, R. J. Johnston, T. J. Melia, T. H. Sollner and J. E. Rothman, J. Cell Biol., 2000, 150, 105117. 